\title{
The Effects of Wall Thickness and Amount of Hardening Agent on the Release Characteristics of Sulfamethoxazole Microcapsules prepared by Gelatin-Acacia Complex Coacervation ${ }^{1)}$
}

\author{
Hrdeo Takenaka, Yoshiaki Kawashima, and Shan Yang Lin \\ Gifu College of Pharmacy ${ }^{2}$
}

(Received June 6, 1979)

\begin{abstract}
The Higuchi model was used to interpret the release characteristics of sulfamethoxazole from gelatin-acacia coacervated microcapsules. For dissolution tests in distilled water and disintegration test solutions No. 1 and 2 (J.P. IX), linear correlations were obtained up to $60-80 \%$ drug release from the microcapsules. In addition, the in vitvo $50 \%$ dissolution time $\left(T_{50}\right)$ was found to correlate with the coating performance, i.e., thickening and hardening of the microcapsule wall. The release rate decreased with increasing wall thickness of the microcapsules, which effectively delayed the release probably by inhibiting or retarding the diffusion rate of the solute into the medium or out from the microcapsule. An increase of formalization with formaldehyde clearly delayed the release rate. The leaching out of the soluble complex from the microcapsules was illustrated by the dissolution profile, and somewhat enhanced the release rate of sulfamethoxazole. The apparent diffusion coefficient of sulfamethoxazole through the microcapsule wall was found to be $1.63 \times 10^{-9}$ to $283 \times 10^{-9} \mathrm{~cm}^{2} / \mathrm{sec}$, and this was a function of coacervation $\mathrm{pH}$ and the amount of formaldehyde used as a hardening agent. Tortuosity in the microcapsule wall was determined to be $0.86 \times 10^{2}$ to $11.4 \times 10^{2}$, based on the dissolution data in distilled water, and was also influenced by coacervation $\mathrm{pH}$ and amount of formaldehyde used.
\end{abstract}

Keywords_-gelatin-acacia complex coacervation; sulfamethoxazole microcapsules; release characteristics; wall thickness; hardening agent; Higuchi model; apparent diffusion coefficient; tortuosity

The increasing use of microcapsules has aroused renewed interest in their better bioavailability and many advantages for clinical use. The various methods and processes employed in in vitro test procedures to obtain prolonged release have been the subject of many reports, patents and reviews. Release of pharmaceuticals from alcohol and salt coacervated gelatin microcapsules was examined in detail by Nixon and co-workers. ${ }^{3}$ ) Although many dissolution data were successfully interpreted by them in terms of the Wagner plot, first-order plot and Higuchi plot, few studies have appeared on the quantitative correlation of drug release with the coating performance of gelatin-acacia coacervated microcapsules. In the previous study by the authors, ${ }^{1)}$ sulfamethoxazole microcapsules were prepared by a gelatin-acacia complex coacervation method and the micromeritic properties were investigated to seek correlations with the preparation conditions. In this paper, the release rate of sulfamethoxazole from gelatin-acacia coacervated microcapsules was determined. The Higuchi model was modified, and an analytical dissolution equation was introduced. In addition, the relationship between the apparent diffusion coefficient through the microcapsule wall and the coacervation $\mathrm{pH}$ or the amount of formaldehyde used for hardening were investigated. The effects of wall thickness on the release rate were successfully analyzed.

1) H. Takenaka, Y. Kawashima, and S.Y. Lin, J. Pharm. Sci., in press.

2) Location: Mitahora-higashi 5-6-1, Gifu, 502, Japan.

3) J.R. Nixon, S.A. Khali1, and J.E. Carless, J. Pharm. Pharmacol., 20, 528 (1968); J.R. Nixon and S.E. Walker, ibid., 23, Suppl. 147S (1971). 


\section{Experimental}

Materials__ The dissolution test samples were gelatin-acacia coacervated microcapsules of sulfamethoxazole prepared at various coacervation $\mathrm{pH}$ values $(2.5$ to 4.0$)$ and with various amounts of formaldehyde $(0$ to $50 \mathrm{ml}) .1)$ Representative micromeritics properties of the microcapsules were as follows: geometric mean diameter, 8.5 to $28.5 \mu \mathrm{m}$; wall thickness, 0.75 to $1.31 \mu \mathrm{m}$; particle density measured with a helium air comparison pycnometer (Micromeritics Inst. Co., model 1302), 1.02 to $1.19 \mathrm{~g} / \mathrm{cm}^{3}$; porosity, 0.158 to 0.277 , as determined from the differences of the particle densities of the microcapsules and the raw materials; other parameters are shown in Table I.1)

Dissolution Test Procedures-The dissolution of sulfamethoxazole from the microcapsules was tested by a modification of the U.S.P. beaker method. An aliquot of $500 \mathrm{ml}$ of distilled water, and medium No. 1 ( $\mathrm{pH}$ 1.2) or No. 2 ( $\mathrm{pH} 7.5$ ) as specified in the disintegration test (J.P. IX, General Test) was used as a dissolution medium. The appropriate amount of the microcapsules, equivalent to $100 \mathrm{mg}$ of sulfamethoxazole, was dispersed into $500 \mathrm{ml}$ of one of the dissolution media in a beaker, which had previously been immersed in a constant temperature bath, and the dissolution medium was allowed to come to $37 \pm 0.5^{\circ}$. The beaker of the dissolution apparatus possessed four baffles and the speed of the agitator, positioned at $2 \mathrm{~cm}$ from the bottom of the beaker, was maintained at $50 \mathrm{rpm}$. Aliquots of $2 \mathrm{ml}$ of the dissolved solution were sampled at the prescribed intervals through a pipette plugged with cotton, and were filtered through a Millipore filter $(3 \mu \mathrm{m})$. Aliquots of the medium (same volume and temperature) were added immediately to the system after each sampling to keep the volume of the dissolution medium constant during the course of the test. A suitable cover was used to prevent loss of the dissolution medium during the experiment. The concentration of dissolved sulfamethoxazole in the medium was determined spectrophotometrically at a suitable ultraviolet region using a double-beam spectrophotometer (Hitachi, model 556).

TABLe I. Micromeritic Properties of G-A Microcapsules ${ }^{1)}$

\begin{tabular}{|c|c|c|c|c|}
\hline Adjusted $\mathrm{pH}$ & $D_{\mathrm{g}}{ }^{1}(\mu \mathrm{m})$ & $l(\mu \mathrm{m})$ & $\rho\left(\mathrm{g} / \mathrm{cm}^{3}\right)$ & $\varepsilon(-)$ \\
\hline 4.0 & 8.5 & 1.31 & 1.02 & 0.270 \\
\hline 3.7 & 15.2 & 1.15 & 1.03 & 0.277 \\
\hline 3.5 & 17.1 & 1.0 & 1.19 & 0.186 \\
\hline 3.0 & 19.1 & 0.87 & 1.08 & 0.217 \\
\hline 2.5 & 28.5 & 0.75 & 1.03 & 0.264 \\
\hline Amount of $\mathrm{HCHO}(\mathrm{ml})$ & $\mathrm{Dg}^{2}(\mu \mathrm{m})$ & & $\rho\left(\mathrm{g} / \mathrm{cm}^{3}\right)$ & $\varepsilon(-)$ \\
\hline 50 & 22.0 & & 1.17 & 0.167 \\
\hline 30 & 20.1 & & 1.11 & 0.203 \\
\hline 15 & 19.0 & & 1.19 & 0.158 \\
\hline 5 & 19.1 & & 1.18 & 0.161 \\
\hline 0 & 17.1 & & 1.19 & 0.186 \\
\hline
\end{tabular}

$D_{\mathrm{g}}{ }^{1}, D_{\mathrm{g}}{ }^{2}:$ mean geometric diameters of unformalized and formalized dried microcapsules, respectively.

$\rho:$ particle density.

$l$ : wall thickness.

$\varepsilon$ : porosity.

\section{Results and Discussion}

\section{Drug Release Behavior from the Microcapsules}

The retarded release profiles of sulfamethoxazole from the gelatin-acacia coacervated microcapsules into the three dissolution media, i.e., distilled water, and disintegration test solutions No. 1 and No. 2 (J.P. IX), are shown in Figs. 1 to 3. The main parameters affecting the drug release behavior from the microcapsules were the type of dissolution medium used, the coacervation $\mathrm{pH}$ and the amount of formaldehyde used in the preparation of the microcapsules. The dissolution medium is known to be a general parameter controlling the dissolution rate of drugs, because the diffusibility of a drug depends greatly on the type of medium used. This may explain the more rapid dissolution in both the disintegration test 


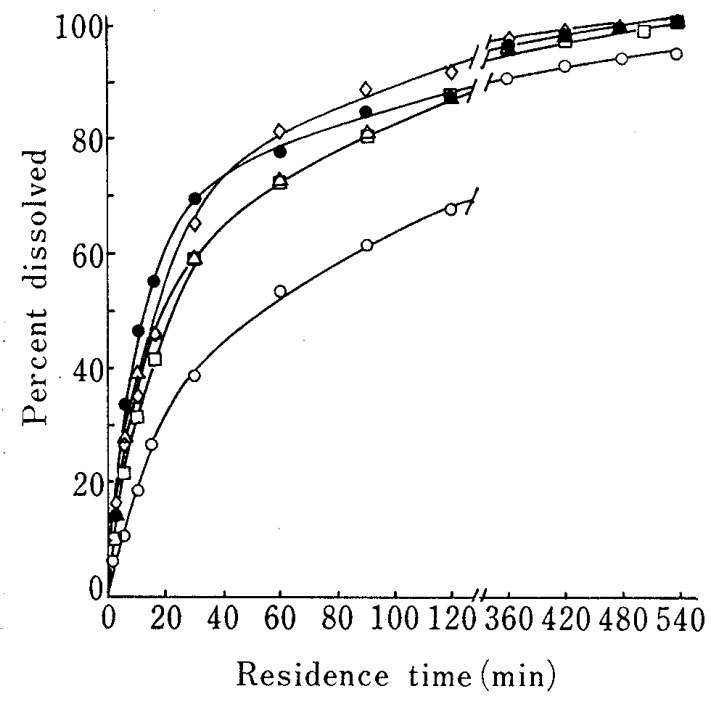

Fig. 1. Dissolution Profile of Sulfamethoxazole from $\mathrm{G}-\mathrm{A}$ Microcapsules in Distilled Water at $37^{\circ}$
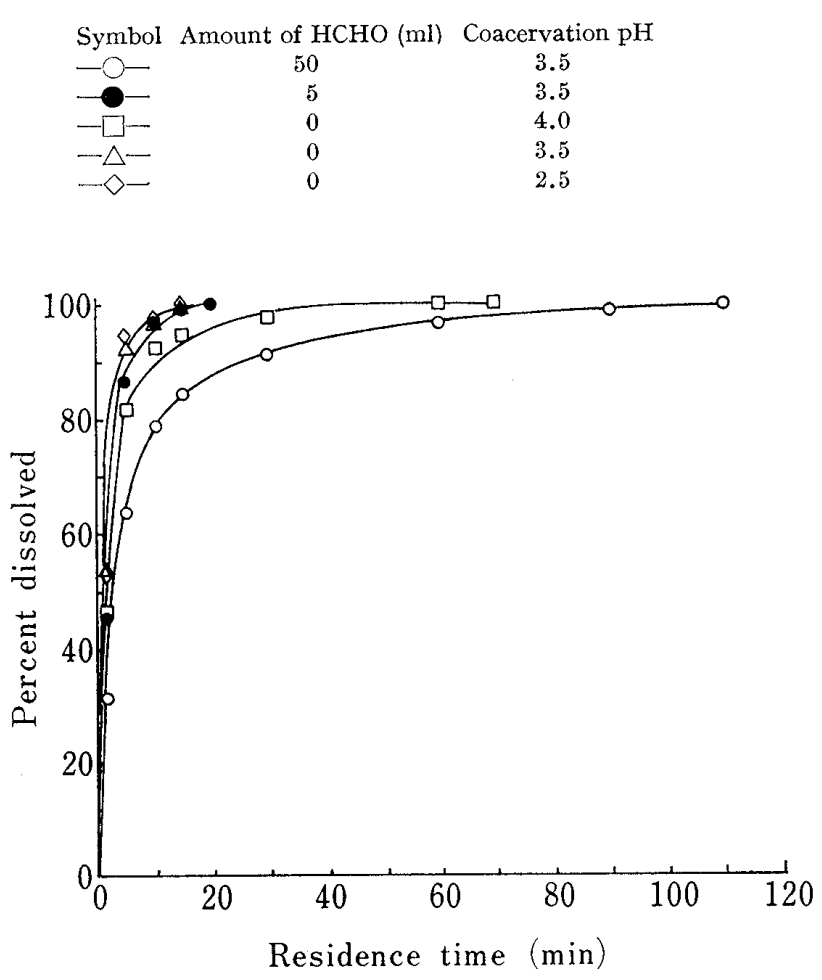

Fig. 3. Dissolution Profile of Sulfamethoxazole from G-A Microcapsules in $\mathrm{pH} 7.5$ Solution at $37^{\circ}$

$\begin{array}{ccc}\text { Symbol } & \text { Amount of } \mathrm{HCHO}(\mathrm{ml}) & \text { Coacervation } \mathrm{pH} \\ -\bigcirc- & 50 & 3.5 \\ -\square & 5 & 3.5 \\ -\square- & 0 & 4.0 \\ -\triangle- & 0 & 3.5 \\ -\diamond- & 0 & 2.5\end{array}$

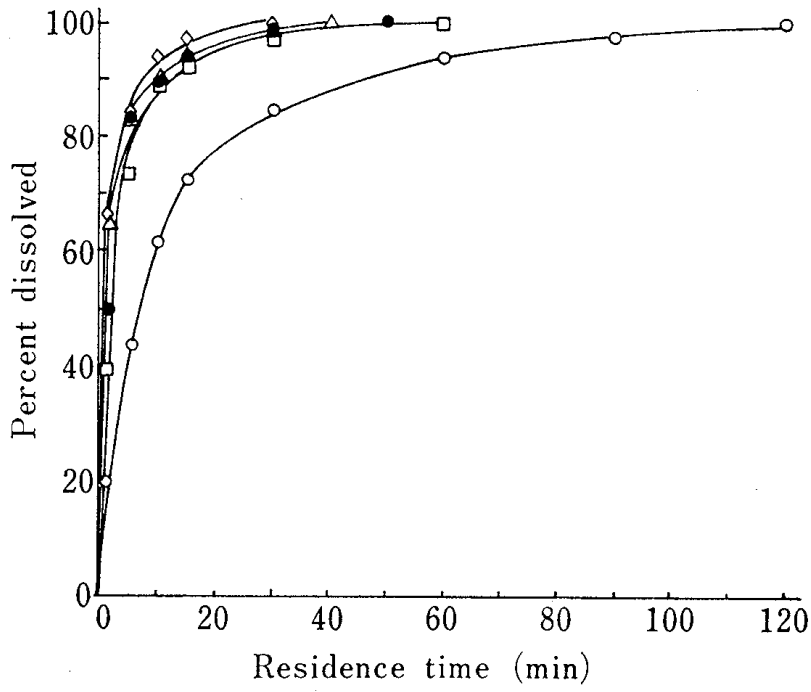

Fig. 2. Dissolution Profile of Sulfamethoxazole from $\mathrm{G}-\mathrm{A}$ Microcapsules in $\mathrm{pH} 1.2$ Solution at $37^{\circ}$

$\begin{array}{ccc}\text { Symbol } & \text { Amount of } \mathrm{HCHO}(\mathrm{ml}) & \text { Coacervation } \mathrm{pH} \\ -\square- & 50 & 3.5 \\ -\square & 5 & 3.5 \\ -\square- & 0 & 4.0 \\ -\triangle- & 0 & 3.5 \\ -\diamond- & 0 & 2.5\end{array}$

solutions $(\mathrm{pH} 1.2$ and $\mathrm{pH}$ 7.5) than in distilled water, since the free sulfamethoxazole may be converted to the salt-form, with improved diffusibility, when the solvent penetrates the microcapsule and contacts the drug. The last two parameters, i.e. the coacervation $\mathrm{pH}$ and the amount of formaldehyde used, may be specific to the present system for controlling the release rate from gelatinacacia coacervated microcapsules. Decrease of coacervation $\mathrm{pH}$ and amount of formaldehyde increased the dissolution rate from the microcapsules in all three dissolution media. In our previous paper, ${ }^{1)}$ it was shown that the wall thickness of the microcapsules decreased successively from 1.31 to $0.75 \mu \mathrm{m}$ with decrease of coacervation $\mathrm{pH}$ from 4.2 to 2.85. This suggests that the $\mathrm{pH}$ effect was due to a change in the diffusibility of the drug through the microcapsule wall. The effect of the amount of formaldehyde

was more pronounced than that of the coacervation $\mathrm{pH}$. Thus, microcapsules formalized with $50 \mathrm{ml}$ of formaldehyde had a release rate one-third of that for microcapsules formalized with $5 \mathrm{ml}$ of formaldehyde in distilled water (Fig. 1). According to the previous paper, in which a net-like rugous structure was demonstrated on the formalized microcapsules, it may be 
suggested that the over-crosslinking effect retards the release of sulfamethoxazole. However, it should be noted that the release rate from the microcapsules formalized with $5 \mathrm{ml}$ of formaldehyde is faster than that of unformalized microcapsules. This is not due to a difference in the surface areas of the two microcapsules, since they had almost the same size, i.e., $17 \mu \mathrm{m}$ and $19 \mu \mathrm{m}$ for the unformalized microcapsules and the microcapsules formalized with $5 \mathrm{ml}$ of formaldehyde, respectively (Table I). The possible formation of a soluble complex of sulfamethoxazole with formaldehyde localized on the microcapsule wall ${ }^{1,3)}$ may explain these peculiar results in the dissolution test. The leaching out of the soluble complex formed on the microcapsule should result in an enhanced release rate of sulfamethoxazole. Although the soluble complex was also formed when other amounts of formaldehyde were used, the strengthened crosslinking of the shell wall effectively delayed the release of sulfamethoxazole from the microcapsules formalized with more than $5 \mathrm{ml}$ of formaldehyde. In the disintegration test solution, the release rate was too fast for the complex formation effect which was detected in distilled water, to be detectable.

The times required for $50 \%$ dissolution of sulfamethoxazole in microcapsules in bulk liquid, i.e. the $50 \%$ dissolution times $\left(\mathrm{T}_{50}\right)$, are plotted against the wall thickness (derived from the coacervation $\mathrm{pH}$ ) and the amount of formaldehyde in Fig. 4. Wagner ${ }^{4}$ suggested that $T_{50}$ is probably the best parameter to correlate dissolution rate with coating performance in vitro, since its value indicates the central tendency of in vitro dissolution data. ${ }^{5} \mathrm{~T}_{50}$ ranged between 0.5 and 6.7 minutes in the disintegration test solutions No. 1 and 2, whereas in distilled water, $T_{50}$ was prolonged to 12 to 52.5 minutes, and the effect of formaldehyde on $T_{50}$ was more marked than in the disintegration test solutions No. 1 and 2.

\section{Analysis of Release Rate using the Higuchi Model}

As described above, wall thickness and the extent of hardening were the factors controlling the sustained release characteristics of the microcapsules. This suggests that diffusion through the gelatin-acacia shell wall is the rate-limiting step, at least in the initial stage of the dissolution, as described by Palmieri. ${ }^{6}{ }$ Higuchi $^{7}$ ) has developed a model to describe the release of medicaments from planar matrix systems in which drug diffusibility is the ratedetermining factor in the release mechanism. The Higuchi model is represented by equation 1 or $\left.2 .{ }^{7}\right)$

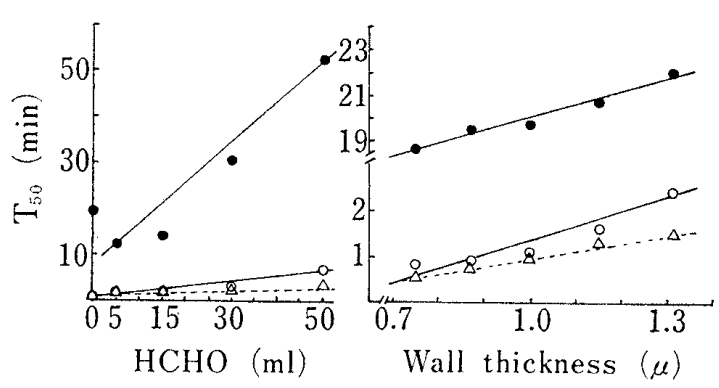

Fig. 4. $50 \%$ Dissolution Time $\left(\mathrm{T}_{50}\right)$ as a Function of Wall Thickness and Amount of $\mathrm{HCHO}$ consumed

Dissolution medium: distilled water; 0 , disintegration solution No. $1(\mathrm{pH} 1.2) ; \triangle$, disintegration solution No. 2 (pH 7.5).

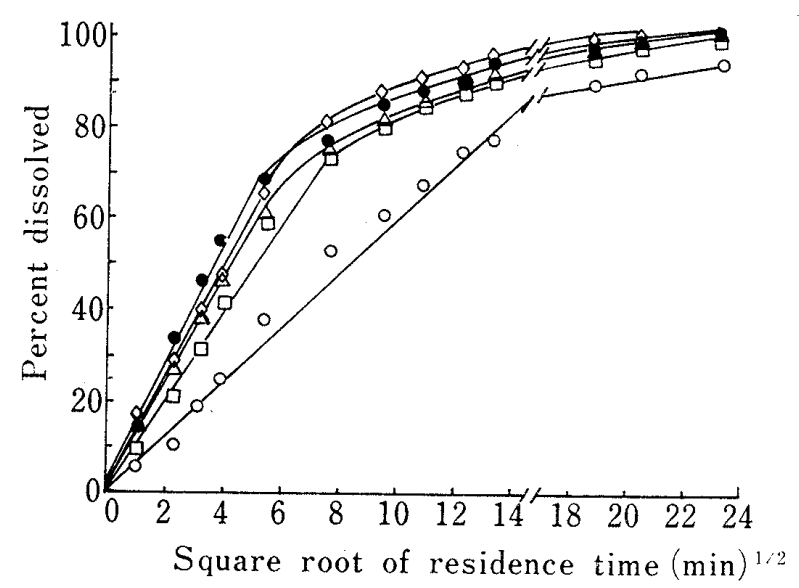

Fig. 5. Drug (\%) dissolved as a Function of the Square Root of Residence Time (Test of the Higuchi Model)

$\begin{array}{ccc}\text { Symbol } & \text { Amount of } \mathrm{HCHO}(\mathrm{ml}) & \text { Adjusted } \mathrm{pH} \\ -0- & 50 & 3.5 \\ & 5 & 3.5 \\ \square- & 0 & 4.0 \\ -\triangle- & 0 & 3.5 \\ - & 0 & 2.5\end{array}$




$$
\begin{aligned}
& \frac{d Q}{d t}=\frac{1}{2} \cdot\left(D\left(2 A-C_{\mathrm{s}}\right) C_{\mathrm{s}} / t\right)^{1 / 2} \\
& Q=\left(D\left(2 A-C_{\mathrm{s}}\right) C_{\mathrm{s}} t\right)^{1 / 2}
\end{aligned}
$$

where $Q$ is the amount absorbed at time $t$ per unit area of exposure $\left(\mathrm{g} / \mathrm{cm}^{2}\right), A$ is the total amount of drug present in the matrix per unit volume $\left(\mathrm{g} / \mathrm{cm}^{3}\right), C_{\mathrm{s}}$ is the solubility of the drug in the external phase of the matrix $\left(\mathrm{g} / \mathrm{cm}^{3}\right)$, and $D$ is the diffusion constant $\left(\mathrm{cm}^{2} / \mathrm{sec}\right)$. When $C_{\mathrm{s}} \ll A$, equation 2 can be simplified to equation 3 .

$$
Q=\left(2 A D C_{\mathrm{s}} t\right)^{1 / 2}
$$

Both sides of equation 3 can be multiplied by $100 \cdot S / V \cdot C_{e}$, where $S$ is the total surface area of microcapsules $\left(\mathrm{cm}^{2}\right), V$ is the volume of the bulk liquid $\left(\mathrm{cm}^{3}\right)$ and $C_{\mathrm{e}}$ is the equilibrium solute concentration in the bulk liquid $\left(\mathrm{g} / \mathrm{cm}^{3}\right)$, leading to equation 4 ,

$$
C_{\mathrm{r}}=100 \cdot S_{\mathrm{v}}\left(2 D C_{\mathrm{s}} t / A\right)^{1 / 2}
$$

where $C_{\mathrm{r}}$ is the percent of the drug dissolved, and $S_{\mathrm{v}}$ is the specific surface area $\left(\mathrm{cm}^{2} / \mathrm{cm}^{3}\right)$. Based on equation 4, the released sulfamethoxazole (\%) was plotted as a function of the square root residence time. For all the dissolution tests, linear correlations were obtained up to $60-80 \%$ drug release, in accordance with the data of many investigators. ${ }^{8)}$ As a representative plot, the release data in distilled water are illustrated in Fig. 5. Once 60$80 \%$ of sulfamethoxazole had been released, the plots deviated from lineality and curved down. This retardation behavior could be explained by the combined effects of swelling of the shell wall of the microcapsules ${ }^{9}$ and an expanding region without solid drug in the microcapsules created by the drug release. ${ }^{10)}$ The swelling may lengthen the diffusion path of a solute molecule in the shell wall appreciably, and after the dissolution of most of the drug, say about $80 \%$, the surface area of the undissolved drug may decrease rapidly, retarding the release rate.

\section{Effect of Coacervation pH and Amount of Formaldehyde on the Diffusion Coefficient through the Microcapsule Wall}

The slope, $\tan \alpha$, of the linear plots at the initial residence time, where $0<C_{\mathrm{r}}<0.6$ to 0.8 , as shown in Fig. 5 , is represented by equation 5 .

$$
\tan \alpha=100 \cdot S_{\mathrm{v}}\left(2 D C_{\mathrm{s}} / A\right)^{1 / 2}
$$

The apparent diffusion coefficient of sulfamethoxazole from the various microcapsules was calculated by using the slopes tabulated in Table II together with equation 5 . The results obtained are listed in Table III, which shows that the apparent diffusion coefficient of sulfamethoxazole in the alkaline medium was larger than in distilled water or the acidic medium, as expected from the dissolution profiles (Fig. 1-3). The plots shown in Fig. 6-A and B reveal a correlation between the apparent diffusion coefficient of sulfamethoxazole and the coacervation $\mathrm{pH}$ or the amount of formaldehyde. The apparent diffusion coefficient decreased

4) J.G. Wagner, "Biopharmaceuticals and Relevent Pharmacokinetics," Drug Intelligence Publications, Hamilton, 1971, p. 213.

5) P.L. Madan, J.C. Price, and L.A. Luzzi, "Microencapsulation: Process and Application," ed. by J.E. Vandegaer, Plenum Press, New York, 1974, p. 39.

6) Anthony Palmieri III, Drug Develop. Ind. Pharm., 3, 309 (1977); idem, Can. J. Pharm. Sci., 12, 88 (1977).

7) T. Higuchi, J. Pharm. Sci., 52, 1145 (1963); idem, ibid., 50, 874 (1961).

8) S. Borodkin and F.E. Tucker, J. Pharm. Sci., 63, 1359 (1974): F.W. Goodhart, R.H. McCoy, and F.C. Ninger, ibid., 63, 1746 (1974).

9) N. Yoshida and C. Thies, J. Colloid Interface Sci., 24, 29 (1967).

10) P.L. Madan, "Microencapsulation: New Techniques and Applications," ed. by T. Kondo, Techno Inc., Tokyo, 1979, p. 11. 
almost linearly with increasing coacervation $\mathrm{pH}$. A linear correlation was also found between the apparent diffusion coefficient and the amount of formaldehyde in the distilled water medium. This was not quite the case for the disintegration test solutions, but the variations can reasonably be explained in the following way. In the acidic medium, a decomplexation of the formaldehyde-gelatin complex making up the microcapsule wall may have occurred, and this may reduce the effect of formalization on the release rate. In the alkaline medium, the gelatin shell of the microcapsules is thickened or solidified. ${ }^{11)}$ This phenomenon may be reflected by a concave curve of the apparent diffusion coefficient plotted against the amount of formaldehyde used.

TABLE II. The Slope of the Linear Plot of Amount of Drug dissolved (\%) against the Square Root of Time

\begin{tabular}{cccc}
\hline & & \multicolumn{2}{c}{ Disintegration test solution } \\
Adjusted pH & Distilled water & No. 1 & No. 2 \\
& & 28.2 & 29.2 \\
4.0 & 10.8 & 35.0 & 40.9 \\
3.7 & 10.8 & 36.7 & 41.5 \\
3.5 & 11.2 & 36.6 & 42.0 \\
3.0 & 11.2 & 36.5 & 42.2 \\
2.5 & 11.5 & & \\
& & & No. 2 \\
\hline & & Disintegration test solution \\
Amount of & Distilled water & No. 1 & 25.1 \\
& & 18.9 & 34.9 \\
\hline 50 & 6.34 & 28.2 & 37.9 \\
30 & 9.33 & 36.7 & 38.7 \\
\hline 5 & 11.7 & 36.7 & \\
\hline
\end{tabular}

TABLE IIT. Effects of Adjusted $\mathrm{pH}$ and Amount of HCHO on the Apparent Diffusion Coefficient and Tortuosity in Distilled Water at $37^{\circ}$

\begin{tabular}{|c|c|c|c|c|}
\hline Adjusted $\mathrm{pH}$ & $D^{*}\left(\mathrm{~cm}^{2} / \mathrm{sec}\right)$ & $l(\mu)$ & $\varepsilon$ & $\tau$ \\
\hline 4.0 & $1.63 \times 10^{-9}$ & 1.31 & 0.270 & $11.38 \times 10^{2}$ \\
\hline 3.7 & $6.28 \times 10^{-9}$ & 1.15 & 0.277 & $3.03 \times 10^{2}$ \\
\hline 3.5 & $11.93 \times 10^{-9}$ & 1.00 & 0.186 & $1.07 \times 10^{2}$ \\
\hline 3.0 & $8.16 \times 10^{-9}$ & 0.87 & 0.217 & $1.83 \times 10^{2}$ \\
\hline 2.5 & $21.06 \times 10^{-9}$ & 0.75 & 0.264 & $0.86 \times 10^{2}$ \\
\hline Amount of $\mathrm{HCHO}$ & al) $D^{*}\left(\mathrm{~cm}^{2} / \mathrm{sec}\right)$ & & $\varepsilon$ & $\tau$ \\
\hline 50 & $4.52 \times 10^{-9}$ & & 0.167 & $2.54 \times 10^{2}$ \\
\hline 30 & $7.09 \times 10^{-9}$ & & 0.203 & $1.97 \times 10^{2}$ \\
\hline 15 & $12.38 \times 10^{-9}$ & & 0.158 & $0.88 \times 10^{2}$ \\
\hline 5 & $14.74 \times 10^{-9}$ & & 0.161 & $0.75 \times 10^{2}$ \\
\hline 0 & $11.93 \times 10^{-9}$ & & 0.186 & $1.07 \times 10^{2}$ \\
\hline
\end{tabular}


(A)

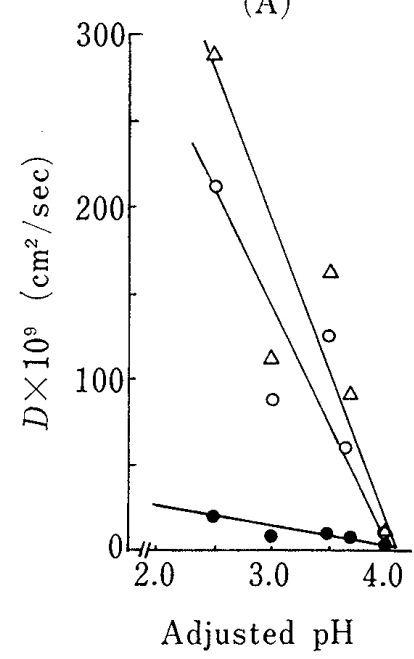

(B)

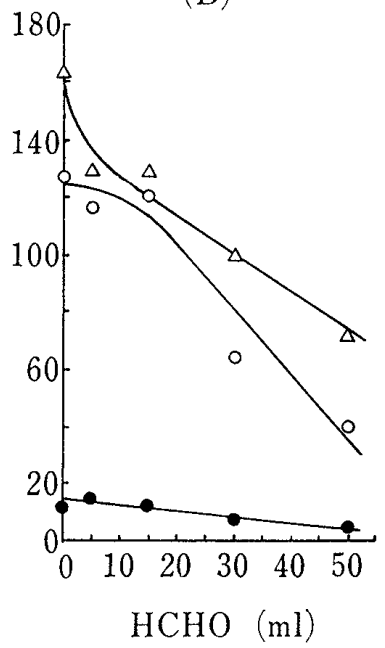

Fig. 6. Correlations of Apparent Diffusion Coefficient with Adjusted $\mathrm{pH}$ and Amount of $\mathrm{HCHO}$ at $37^{\circ}$

Dissolution medium: 0 , distilled water; $\bigcirc$, disintegration solution No. $1(\mathrm{pH} 1.2) ; \triangle$, disintegration solution $\mathrm{N}_{\mathrm{O}} .2(\mathrm{pH}$ 7.5).

The apparent diffusion coefficient can be described as a function of tortuosity $(\tau)$ and porosity $(\varepsilon)$ in the microcapsule as in equation 6 ,

$$
D=\frac{D^{*} \varepsilon}{\tau}
$$

where $D^{*}$ is the intrinsic diffusion coefficient. The diffusion coefficient of raw sulfamethoxazole in water was calculated by Nogami ${ }^{12}$ ) using the Arnold equation. ${ }^{13)}$ At $20^{\circ}$ the diffusion coefficient was $5.21 \times 10^{-6}\left(\mathrm{~cm}^{2} / \mathrm{sec}\right)$. The diffusion coefficient at $37^{\circ}$, the temperature at which the dissolution test was undertaken in the present study, can be estimated from equation $7 .{ }^{14)}$

$$
D_{\mathrm{t}}=\frac{D_{20}}{1.6}(1+0.03 t)
$$

At $37^{\circ}$ in a water medium, the diffusion coefficient of sulfamethoxazole $\left(D_{37}\right)$ is $6.87 \times 10^{-6}$ $\left(\mathrm{cm}^{2} / \mathrm{sec}\right)$. The tortuosity can be calculated by inserting the porosity data given in Tables I and III and $D_{37}$ into equation 6 . There appeared to be an increase in tortuosity of the shell wall of the microcapsules with increasing $\mathrm{pH}$. The relationship between wall coating and tortuosity is shown in Fig. 7. This indicates that a higher tortuosity is presented in a thicker wall. Moreover, increase in tortuosity was also found with increasing amount of hardening agents. It is clear that the extent of hardening also influences the tortuosity, which plays an important role in the dissolution characteristics.

Acknowledgement and Correspondence The authors acknowledge the valuable advice of Dr. B.A. Matthews (Ciba-Geigy Co., Jakarta, Indonesia) in the preparation of the text. Inquiries should be directed to X. Kawashima.

11) G.L. Jenkins, D.E. Francke, E.A. Brecht, and G.J. Sperandio, "The Art of Compounding," The Blakiston Co., New York, 1952, p. 479.

12) H. Nogami, T. Nagai, and A. Suzuki, Chem. Pharm. Bull. (Tokyo), 14, 329 (1966).

13) J.H. Arnold, J. Am. Chem. Soc., 52, 2937 (1930).

14) K. Sato, "Bussei Teisu Suisanho," Maruzen, Tokyo, 1957, p. 306. 